\title{
Supernova remnants, planetary nebulae and the distance to NGC 4214
}

\author{
Michael A. Dopita · Daniela Calzetti • Jesús Maíz Apellániz • William P. Blair · \\ Knox S. Long • Max Mutchler • Bradley C. Whitmore • Howard E. Bond • \\ John MacKenty • Bruce Balick • Marcella Carollo • Michael Disney • Jay A. Frogel • \\ Robert O'Connell • Donald Hall • Jon A. Holtzman • Randy A. Kimble • \\ Patrick McCarthy · Francesco Paresce • Abhijit Saha • Alistair R. Walker • Joe Silk • \\ Marco Sirianni · John Trauger · Rogier Windhorst • Erick Young
}

Received: 7 February 2010 / Accepted: 23 March 2010 / Published online: 13 April 2010

(C) Springer Science+Business Media B.V. 2010

\begin{abstract}
We present narrow band, continuum subtracted $\mathrm{H} \alpha,[\mathrm{S} \mathrm{II}], \mathrm{H} \beta,[\mathrm{O} \mathrm{III}]$ and [O II] data taken with the Wide Field Camera 3 on the Hubble Space Telescope in the nearby dwarf starburst galaxy NGC 4214. From these images, we identify seventeen new planetary nebula candidates, and seven supernova remnant candidates. We use the observed emission line luminosity function of the planetary nebulae to establish a new velocity-independent distance to NGC 4214. We conclude that the PNLF technique gives a reddening independent distance to NGC 4214 of $3.19 \pm 0.36 \mathrm{Mpc}$, and that our current best-estimate of the distance to this galaxy ids $2.98 \pm 0.13 \mathrm{Mpc}$.
\end{abstract}

\footnotetext{
M.A. Dopita $(\bowtie)$

Research School of Astronomy \& Astrophysics, The Australian National University, Weston Creek, ACT 2611, Australia

e-mail: michael.dopita@anu.edu.au

D. Calzetti

University of Massachusetts, Amherst, MA 01003, USA

J. Maíz Apellániz

Instituto de Astrofísica de Andalucía-CSIC, Granada, Spain

W.P. Blair

Johns Hopkins University, Baltimore, MD, USA

K.S. Long · M. Mutchler · B.C. Whitmore · H.E. Bond · J. MacKenty

Space Telescope Science Institute, Baltimore, MD, USA

B. Balick

University of Washington, Seattle, WA, USA

M. Carollo

Institute of Astronomy, ETH-Zurich, Zurich, Switzerland
}

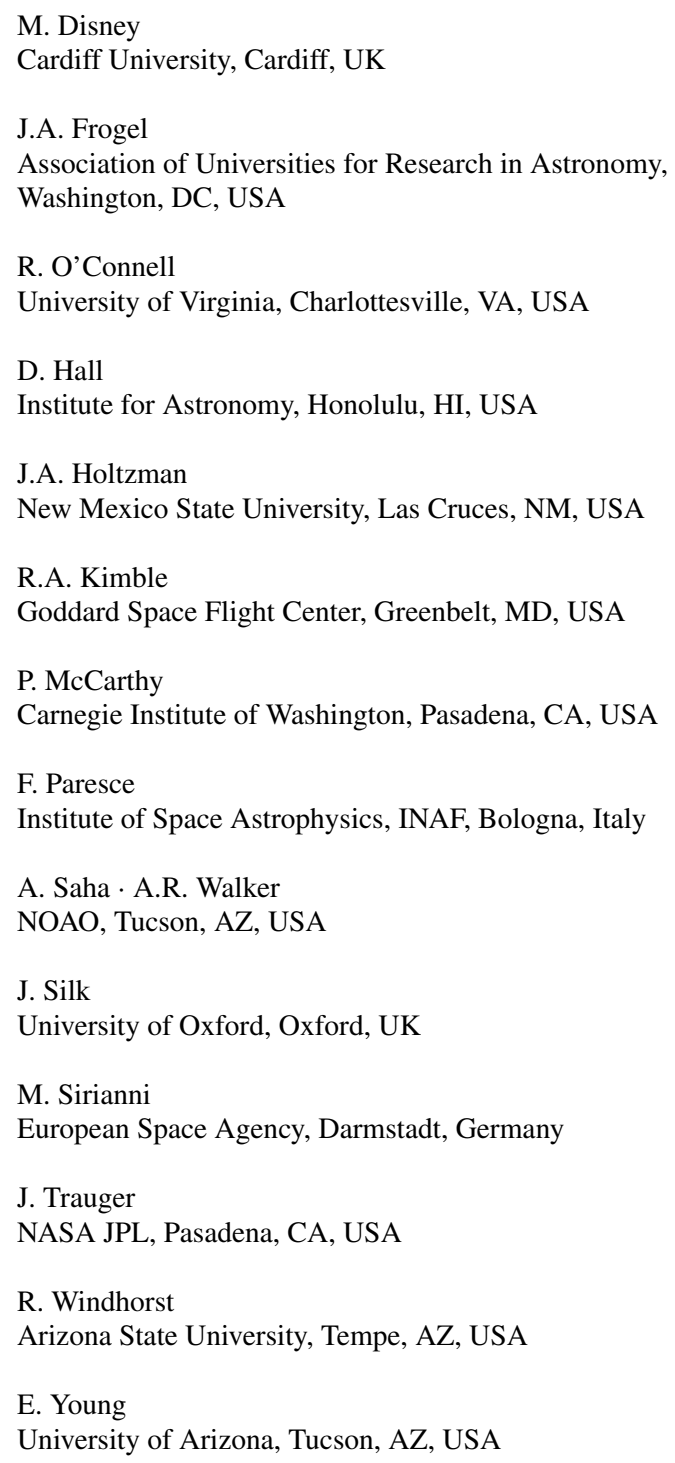


Keywords Supernovae: general - ISM: structure, supernova remnants $\cdot$ Stars: planetary nebulae $\cdot$ Galaxies: ISM, starburst, structure

\section{Introduction}

NGC 4214 is a nearby Magellanic star bursting dwarf irregular galaxy. It has several intense star-forming regions concentrated along the bar of the galaxy (MacKenty et al. 2000; Maíz-Apellániz et al. 2002). According to the NASA/IPAC Extragalactic Database (NED), its heliocentric radial velocity, $v_{\text {hel }}=291 \mathrm{~km} \mathrm{~s}^{-1}$, it covers about 9 arc min on the sky, and has the classification $\mathrm{IAB}(\mathrm{s}) \mathrm{m}$. Its metallicity has been measured indirectly by Ubeda et al. (2007) using the ratio of the blue to red supergiant stars and directly by Kobulnicky and Skillman (1996) who obtained $12+\log (\mathrm{O} / \mathrm{H})=8.21-$ 8.36 , with little evidence of any abundance gradient. This value can be compared to the Magellanic Clouds. Russell and Dopita (1992) obtained $12+\log (\mathrm{O} / \mathrm{H})=8.35$ for the interstellar medium in the $\mathrm{LMC}$ and $12+\log (\mathrm{O} / \mathrm{H})=$ 8.03 for the SMC, while the planetary nebulae yield $12+$ $\log (\mathrm{O} / \mathrm{H})=8.41$ for the $\mathrm{LMC}$ and $12+\log (\mathrm{O} / \mathrm{H})=8.05$ for the SMC (Dopita and Meatheringham 1991a, 1991b; Dopita et al. 1997). Clearly the Magellanic Clouds provide useful references for comparative studies.

In this paper we present new narrow-band imaging results in this galaxy obtained with the Wide Field Camera 3 (WFC3) on the Hubble Space Telescope (HST). These enable us to both identify the supernova remnants (SNR) and to study their morphology. We can also identify planetary nebulae (PN) and use the photometry of these to derive a distance to NGC 4214. In Sect. 2 we describe the data and the data reduction, in Sect. 3 the identification of the PN, in Sect. 4 the identification and investigation of the SNR, and in Sect. 5 we use the observed planetary nebula luminosity function (PNLF) to establish a new velocity-independent distance to NGC 4214.

\section{WFC3 observations}

\subsection{The WFC3 dataset}

The WFC3 data were obtained as part of WFC Science Oversight Committee (SOC) Early Release Science (ERS) program (program ID11360: Principal Investigator: Robert O'Connell, The University of Virginia). The NGC 4214 field is centered at $\mathrm{RA}=12: 15: 38.9 \mathrm{Dec}=+36: 19: 47(\mathrm{~J} 2000)$. Either three or four dithered exposures were taken in each narrow-band filter to remove cosmetic defects and assist in the cosmic ray removal. For this paper a sub-set of the data taken in the UVIS channel has been used. The observing log for these observations is given in Table 1.
Table 1 Log of WFC3 observations (Prop ID\# 11360)

\begin{tabular}{lll}
\hline Filter: & Exp(s) & Date \\
\hline F336W & $3 \times 424+400$ & $2009-12-23$ \\
F373N & $2 \times 450+360$ & $2009-12-25$ \\
F438W & $3 \times 385+375$ & $2009-12-22$ \\
F487N & $3 \times 425+485$ & $2009-12-24$ \\
F502N & $3 \times 490$ & $2009-12-24$ \\
F547M & $4 \times 425$ & $2009-12-24$ \\
F657N & $3 \times 385+437$ & $2009-12-23$ \\
F673N & $4 \times 735$ & $2009-12-25$ \\
F814W & $3 \times 348+285$ & $2009-12-24$ \\
\hline
\end{tabular}

\subsection{Reduction of observations}

The data was passed through the MultiDrizzle software (Fruchter et al. 2009; Fruchter and Hook 2002) which produces a combined,cosmic ray cleaned and distortion corrected data product with a built-in world coordinate system. For the observations with only three temporally grouped exposures per filter (F373M, F502N) cosmic ray events cannot be fully cleaned from the 'chip gap' region across the center of the field.

The mean redshift of NGC 4214 is only $291 \mathrm{~km} \mathrm{~s}^{-1}$. This is sufficiently small to ensure that the line emission is not significantly shifted from the peak of the narrow-band filter bandpasses. The filter bandpasses are also sufficiently wide to ensure that any likely velocity dispersion in SNR shells $\left(\sim 200-400 \mathrm{~km} \mathrm{~s}^{-1}\right)$ is also comfortably accommodated.

There is a small offset between images taken at different visits to the same target. These were removed using the imalign task in the IRAF package. Two weighted wide-band images were combined and scaled to provide a continuum reference image for each narrow-band image; F336W and F438W for the [O II] data, F438W and F555W for the $\mathrm{H} \beta$ and [O III] images and F555W and F814W for the $\mathrm{H} \alpha$ and [S II] images. The weighting of the broadband images was determined by the offset of their bandpass in logarithmic wavelength with respect to the emission line in question.

Each scaled continuum image was subtracted from the relevant line image to produce a pure emission line image. The subtraction is not perfect, and In some cases, the mean continuum image was re-scaled, and re-subtracted from the emission line image to optimally remove the stellar residuals. Despite this process, under-subtracted and oversubtracted residuals of extremely red or blue stars still remain in our emission line images.

The result of this exercise is a set of "pure" emission line images in [O II] $\lambda 3737,9, \mathrm{H} \beta,[\mathrm{O}$ III] $\lambda 5007, \mathrm{H} \alpha+[\mathrm{N} \mathrm{II}]$ $\lambda 6548,84$ and $[\mathrm{S} \mathrm{II}] \lambda 6717,31$. The most successful combination to produce a color image proves to be one related to excitation of the gas, and is shown in Fig. 1. In this image, 


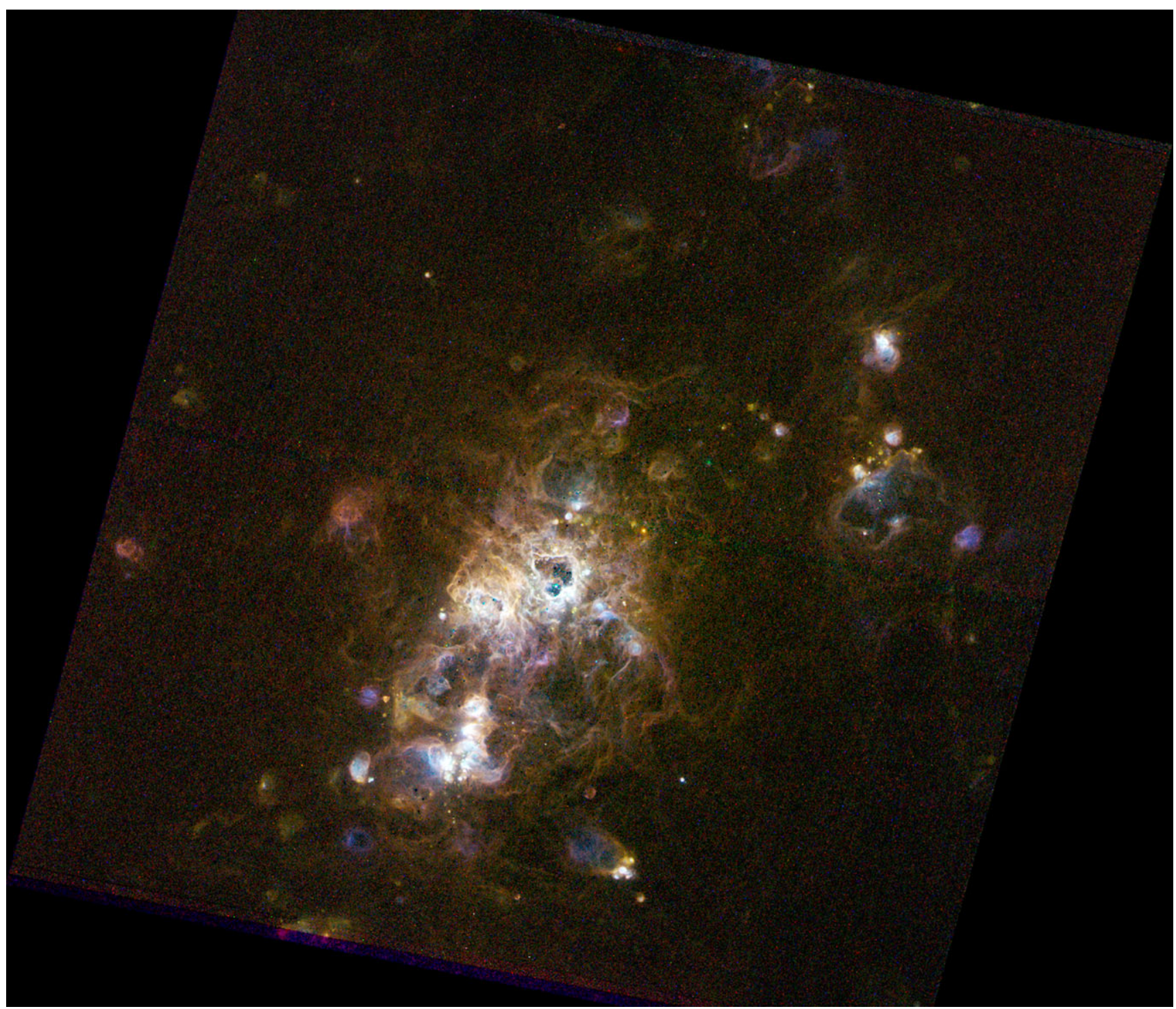

Fig. 1 A color image of the emission line gas in NGC 4214. North is at the top, and east at the left. The red channel consists of the mean of the low-excitation species, [O II] and [S II], the green channel is $\mathrm{H} \alpha$ and shows all ionized gas, while the blue channel is the high-excitation species [O III]. Thus the color is related to the local excitation of the ionized plasma. The stretch is chosen to be square root to bring out the fainter features supernova remnants and diffuse, low excitation gas appears red or gold, normal high excitation H II regions appear blue or turquoise, and are often surrounded by reddish ionization fronts. A few ionized regions are very high excitation, and appear blue in this image. These may well be associated with Wolf-Rayet central stars.

\section{Identification of planetary nebulae}

Planetary nebulae $(\mathrm{PN})$ are characterized by very strong [O III] emission relative to the Balmer lines, while [O II] and [S II] are generally much weaker. In addition, at the distance of NGC 4214 we expect PN to appear as unresolved, stellar-like objects. We therefore identified them by separately blinking either [O III] and [S II] and $\mathrm{H} \alpha$ images, or the $\mathrm{H} \beta$, [O III] and $\mathrm{H} \alpha$ combination. This allows us to clearly distinguish the PN from any confusing cosmic ray residuals, or unresolved compact $\mathrm{H}$ II regions.

Once identified, we measured the $\mathrm{H} \alpha$, [O III $] \lambda 5007$ and $\mathrm{H} \beta$ fluxes of these $\mathrm{PN}$ using the digiphot aperture photometry routines in IRAF. The photometric conversion from counts per sec to erg $\mathrm{cm}^{-2} \mathrm{~s}^{-1}$ has been established by the on-orbit calibration. These conversions are $2.165 \times 10^{-16}$ for the F657N filter $2.891 \times 10^{-16}$ for the F487N filter and $2.88 \times 10^{-16}$ for the F502N filter. For a few PN, the signal 
Table 2 The Positions and line fluxes of the NGC 4214 Planetary Nebula Candidates

\begin{tabular}{llllll}
\hline Name & RA & Dec $(\mathrm{J} 2000)$ & $F_{5007}$ & $F_{\mathrm{H} \alpha}$ & $F_{\mathrm{H} \beta}$ \\
\hline NGC4214 - PN1 & $12: 15: 34.450$ & $+36: 19: 37.01$ & $2.63 \mathrm{E}-16$ & $1.33 \mathrm{E}-16$ & \\
NGC4214 - PN2 & $12: 15: 35.450$ & $+36: 20: 47.50$ & $6.10 \mathrm{E}-16$ & $2.36 \mathrm{E}-16$ & $7.52 \mathrm{E}-17$ \\
NGC4214 - PN3 & $12: 15: 35.284$ & $+36: 19: 14.24$ & $5.48 \mathrm{E}-16$ & $1.38 \mathrm{E}-16$ & $5.78 \mathrm{E}-17$ \\
NGC4214 - PN4 & $12: 15: 36.637$ & $+36: 19: 15.19$ & $7.00 \mathrm{E}-16$ & $1.76 \mathrm{E}-16$ & \\
NGC4214 - PN5 & $12: 15: 36.919$ & $+36: 19: 55.03$ & $5.06 \mathrm{E}-16$ & $2.89 \mathrm{E}-16$ & $4.63 \mathrm{E}-17$ \\
NGC4214 - PN6 & $12: 15: 37.505$ & $+36: 20: 02.16$ & $6.51 \mathrm{E}-16$ & $3.34 \mathrm{E}-16$ & \\
NGC4214 - PN7 & $12: 15: 38.475$ & $+36: 20: 18.00$ & $1.11 \mathrm{E}-15$ & $3.08 \mathrm{E}-16$ & $1.12 \mathrm{E}-16$ \\
NGC4214 - PN8 & $12: 15: 38.757$ & $+36: 20: 10.24$ & $3.22 \mathrm{E}-16$ & $1.29 \mathrm{E}-16$ & $4.63 \mathrm{E}-17$ \\
NGC4214 - PN9 & $12: 15: 38.787$ & $+36: 19: 26.52$ & $6.37 \mathrm{E}-16$ & $2.23 \mathrm{E}-16$ & $4.63 \mathrm{E}-17$ \\
NGC4214 - PN10 & $12: 15: 38.855$ & $+36: 19: 21.73$ & $3.15 \mathrm{E}-15$ & $1.54 \mathrm{E}-15$ & $3.82 \mathrm{E}-16$ \\
NGC4214 - PN11 & $! 2: 15: 39.062$ & $+36: 18: 51.42$ & $1.30 \mathrm{E}-16$ & $5.41 \mathrm{E}-17$ & $1.89 \mathrm{E}-17$ \\
NGC4214 - PN12 & $12: 15: 39.914$ & $+36: 19: 21.61$ & $6.37 \mathrm{E}-16$ & $3.55 \mathrm{E}-16$ & $1.10 \mathrm{E}-16$ \\
NGC4214 - PN13 & $12: 15: 40.061$ & $+36: 19: 11.00$ & $9.71 \mathrm{E}-16$ & $1.40 \mathrm{E}-15$ & $2.54 \mathrm{E}-16$ \\
NGC4214 - PN14 & $12: 15: 40.972$ & $+36: 20: 17.53$ & $5.55 \mathrm{E}-16$ & $1.45 \mathrm{E}-16$ & $5.49 \mathrm{E}-17$ \\
NGC4214 - PN15 & $12: 15: 43.784$ & $+36: 19: 47.78$ & $2.45 \mathrm{E}-16$ & $1.73 \mathrm{E}-16$ & $6.36 \mathrm{E}-17$ \\
NGC4214 - PN16 & $12: 15: 45.748$ & $+36: 19: 42.00$ & $4.80 \mathrm{E}-16$ & $1.61 \mathrm{E}-16$ & \\
NGC4214 - PN17 & $12: 15: 46.207$ & $+36: 19: 01.52$ & $1.50 \mathrm{E}-16$ & $1.34 \mathrm{E}-16$ & \\
\hline Typical error: & & & $\pm 2.3 \mathrm{E}-17$ & $\pm 1.1 \mathrm{E}-17$ & $\pm 2.3 \mathrm{E}-17$ \\
\hline
\end{tabular}

to noise was too low to permit a reliable measurement of the $\mathrm{H} \beta$ flux.

The PN candidate positions and fluxes are listed in Table 2. Amongst these candidates, we believe that NGC4214 - PN10 and NGC4214 - PN13 are probably compact highexcitation H II regions. In the case of NGC4214 - PN10 we base this judgement on three facts. First, the measured [O III] flux is considerably larger than any other object. Second, the $\mathrm{H} \alpha$ to $\mathrm{H} \beta$ ratio is appreciably larger than the Case $\mathrm{B}$ recombination value, indicating the presence of dust and third, the object appears to be marginally resolved. NGC4214 - PN13 is the third brightest object in [O III]. It also shows a large Balmer decrement and evidence for a faint resolved extension. Objects such as these probably account for at least some of the luminous "stragglers" found in the [O III] luminosity function found in other, more distant galaxies (Jacoby et al. 1990).

\section{Identification of SNR candidates}

To identify supernova remnant (SNR) candidates in NGC 4214 we have used the classical technique pioneered by Mathewson and Healey (1964), Westerlund and Mathewson (1966) and Mathewson and Clarke (1973). This relies both on the identification with a non-thermal radio source, and at optical wavelengths, on the strength of the $[\mathrm{S} \mathrm{II}] / \mathrm{H} \alpha$ ratio (typically greater than 0.4 , characteristic of the cooling and recombination zone of a radiative shock. Other line emission criteria have been developed from radiative shock wave theory (Dopita 1977a, 1977b) and may also be used. These include a high $[\mathrm{O} \mathrm{II}] / \mathrm{H} \beta$ and a relatively low $[\mathrm{O}$ III $] / \mathrm{H} \beta$ ratio. Techniques based upon this methodology have been successfully used to survey for SNR in the Magellanic Clouds (Lasker 1977; Mathewson et al. 1983, 1984, 1985), in M31 and other local group galaxies (D'Odorico et al. 1980), in M33 (D'Odorico et al. 1978; Blair et al. 1981; Long et al. 1990, 2010; Gordon et al. 1998) and in the southern grand design star-forming galaxy M83 (Blair and Long 2004). More recently, Dopita et al. (2010) have used WFC3 data obtained as part of the WFC3 SOC program to identify 60 supernova remnant candidates, and a handful of young ejecta-dominated candidates in M83.

Candidates in the general field were identified by blinking the aligned and match-scaled $\mathrm{H} \alpha$, [S II] and [O II] images. The SNR candidates appear relatively bright on both the [S II] and [O II] images compared with $\mathrm{H} \alpha$. For these candidates, we could check the SNR identification by direct measurement of the ratio of $[\mathrm{S} \mathrm{II}]$ to $\mathrm{H} \alpha$ taking into account the fact that the HST F657N filter also transmits the [N II] $\lambda 6584$ line. The "classical" definition of a SNR is $[\mathrm{S} \mathrm{II}] / \mathrm{H} \alpha>0.4$. Since the [N II] line may be as strong as $\sim 50 \%$ of $\mathrm{H} \alpha$, we relax the above criterion for identification of a SNR to the condition $F_{\mathrm{F} 673 \mathrm{~N}} / F_{\mathrm{F} 657 \mathrm{~N}}>0.3$. We also checked that the old SNR candidates are characterized by low $[\mathrm{O} \mathrm{III}] / \mathrm{H} \beta$ ratios.

In the case of NGC 4214 we do not have to rely solely on the optical line ratios to identify the SNR candidates, because there has recently been published a sensitive $(\sim 20 \mu \mathrm{Jy})$ radio survey of the galaxy at the wavelengths of 
Table 3 SNR Candidates in NGC 4214

\begin{tabular}{llllll}
\hline Name & RA & Dec (J2000) & $D$ (arc sec.) & Radio ID (CW09) & Nature \\
\hline NGC4214-SNR1 & $12: 15: 38.963$ & $+36: 18: 58.85$ & 1.98 & NGC4214-04 & SNR \\
NGC4214-SNR2 & $12: 15: 40.000$ & $+36: 18: 40.72$ & 1.09 & NGC4214-09 & SNR \\
NGC4214-SNR3 & $12: 15: 40.008$ & $+36: 19: 36.00$ & 2.70 & NGC4214-10 & SNR \\
NGC4214-SNR4 & $12: 15: 40.136$ & $+36: 19: 30.04$ & $0.25 \times 0.4$ & NGC4214-11 & YSNR \\
NGC4214-SNR5 & $12: 15: 41.902$ & $+36: 19: 15.20$ & 1.05 & NGC4214-19 & SNR \\
NGC4214-SNR6 & $12: 15: 42.456$ & $+36: 19: 47.78$ & 2.80 & NGC4214-20 & SNR+HII \\
NGC4214-SNR7 & $12: 15: 45.639$ & $+36: 19: 41.50$ & 2.40 & NGC4214-23 & SNR+HII \\
\hline
\end{tabular}
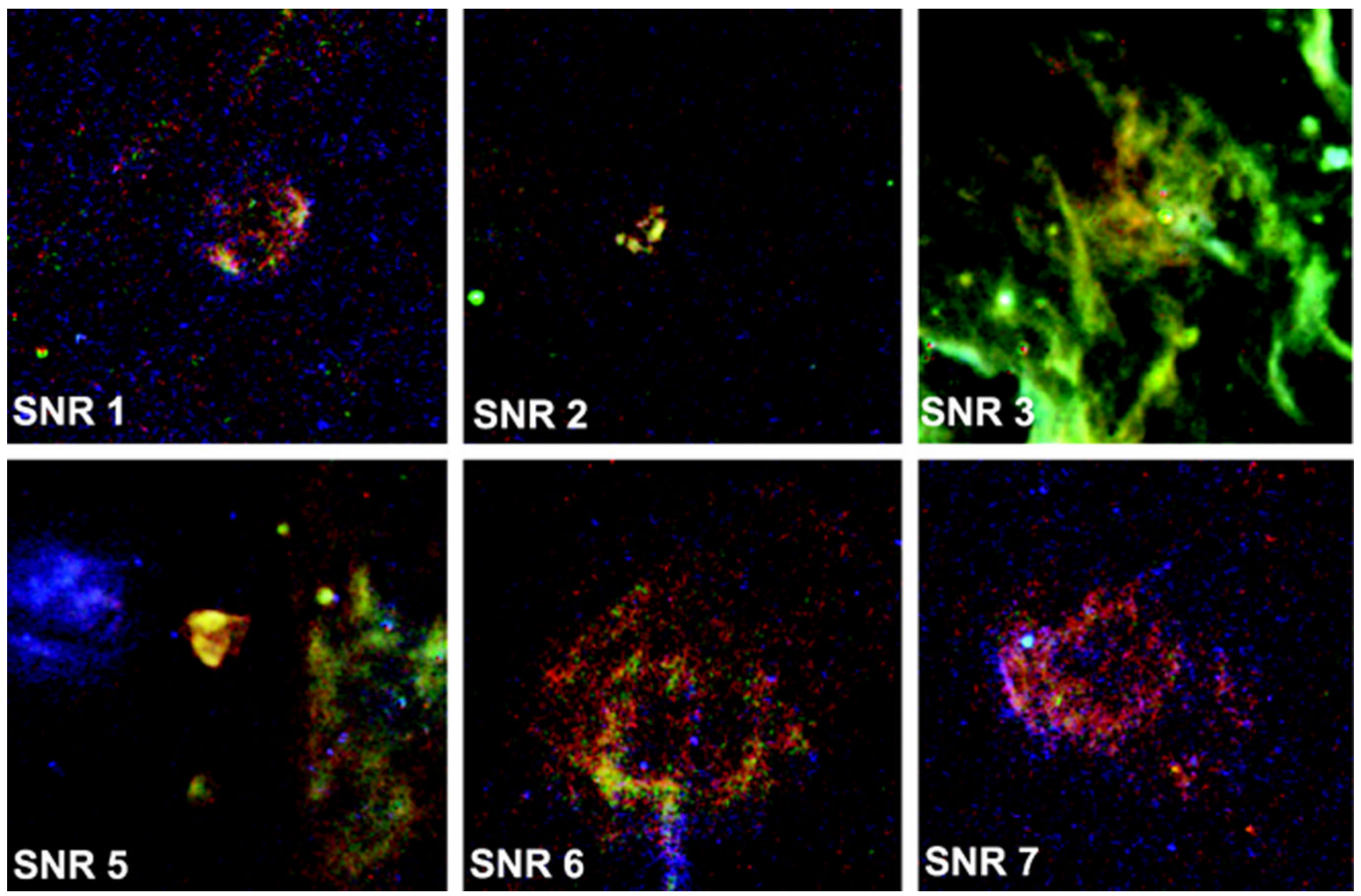

Fig. 2 Color images of the old supernova remnant candidates found in NGC 4214. North is at the top, and east at the left. Each image covers $8 \times 8$ arc sec. The filter combinations making up the RGB images are Red channel [O II] and [S II], Green channel $\mathrm{H} \alpha$ and Blue channel [O III]. Note the unusual "blobby" morphology of SNR 2. SNR

20, 6 and $3.6 \mathrm{~cm}$ (Chomiuk and Wilcots (2009) which we will refer to as CW09). Table 3 gives the positions, angular diameters, CW09 radio identifications and assumed nature of the source (old SNR, young SNR or composite SNR + $\mathrm{H}$ II region). Figure 2 gives color images of the old SNR candidates, and of the composite nature sources.

Several of our supernova remnant candidates had previously been identified as radio sources in the VLA data taken by Eck et al. (2002) as part of a search for radio emission
3 is embedded in very bright photo-ionized nebulosity in the main star forming region. The blue-violet object to the east of the very bright candidate SNR 5 is probably excited by a Wolf-Rayet star. The turquoise stellar-like object in the eastern part of SNR 7 is the planetary nebula candidate number 17 from historical supernova with ages up to about 80 years. The galaxy NGC 4214 was studied because it was the site of the historical supernova SN1945A, which was classified as a Type $\mathrm{Ib}$ event. The position of this supernova is given by Eck et al. (2002) as 12:15:45.85 + 36:16:14.2 (J2000.0). Regrettably, this supernova lies outside the WFC3 field, and so we could not search for any remnant associated with it. However, amongst the radio sources identified by Eck et al. (2002), we find that NGC4214-SNR1 is positionally 
coincident with their source NGC $4214 \delta$ and NGC4214SNR2 corresponds to their source NGC $4214 \pi$. In addition, NGC4214-SNR3 may be NGC $4214 \eta$ and NGC4214-SNR5 could be the radio source NGC $4214 \rho$.

It is also worth discussing the comparison of the CW09 sources, and the sources identified here in the optical. The CW09 sources NGC4214-04, NGC4214-09, NGC4214-10 and NGC4214-19 are most certainly genuine old SNR, and fit all the criteria. NGC4214-10 (our NGC4214-SNR3) is in a very crowded and confused region, but (gold-colored) shock excited filaments are clearly visible in Fig. 2. The sizes estimated by CW09 are generally larger than what we measure, but this may well be simply an issue of the limited resolution of the VLA images.

We find the CW09 source NGC4214-02 (identified as a SNR by them) is coincident with an extremely bright $\mathrm{H}$ II region. There may well be a SNR embedded in it, but if so, we could find no trace of it in the optical line ratios. Likewise, we find no trace of NGC4214-8, NGC4214-12 and NGC4214-18. Indeed, these are not even correlated with any particularly bright nebular features.

The CW09 source NGC4214-11 (our source name NGC4214-SNR4) is a very interesting case. This object certainly does not fit the classical criteria for a SNR listed above, but in [O III] the small elongated central object probably has the highest surface brightness of any ionized region of the galaxy, as is shown in Fig. 3. It is also prominent in both [O II] and $\mathrm{H} \alpha$, but is weak in [S II]. The central object is surrounded by a complex filamentary shell of varying excitation, and the overall morphology is strongly suggestive of a very young oxygen-rich SNR sitting within a massloss bubble blown in the pre-supernova phase. The size of this bubble ( $\sim 2$ arc sec. or $\sim 30 \mathrm{pc})$ is similar to that found around N132D in the LMC (Blair et al. 1981), and is therefore consistent with our hypothesis.

Finally the two sources NGC4214-20 and NGC421423 (our sources NGC4214-SNR6 and NGC4214-SNR7) are large shells embedded in even larger shells. Both of these are classified by CW09 as H II regions on the basis of their flat radio spectra. However, the emission line ratios are consistent with a shock excited origin. These objects may be either old SNR embedded in $\mathrm{H}$ II regions, or else they may simply be old H II regions in which the UV photon output of the star has faded, and the ionized gas is now recombining. Kinematic data would reveal which of these is the correct interpretation.

NGC4214-SNR3 had already been identified as a SNR by MacKenty et al. (2000), where it is referred to as knot IF. Their detection is based on its optical emission line ratios, the steep radio spectral index, and the existence of a high velocity $\sigma=60-65 \mathrm{~km} \mathrm{~s}^{-1}$ component in the optical emission lines (Maíz-Apellániz et al. 1998, 1999).

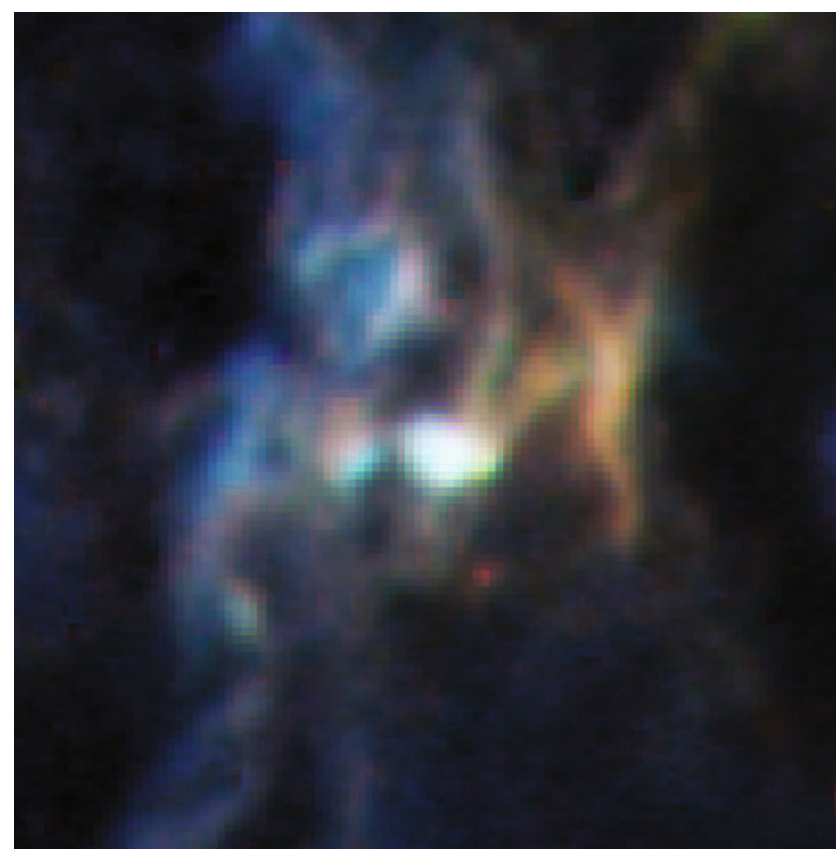

Fig. 3 The source NGC4214-SNR4. North is at the top, and east at the left. This image covers $4 \times 4$ arc sec. The filter combinations making up the RGB images are Red channel [O II] and [S II], Green channel $\mathrm{H} \alpha$, and Blue channel [O III]. The morphology is reminiscent of a young oxygen-rich SNR (identified with the whitish blob elongated in the E-W direction in the center of the field) surrounded by a $\sim 2$ arc sec. $(\sim 30 \mathrm{pc})$ diameter mass-loss bubble originating from the pre-supernova star

NGC4214-SNR4 corresponds to the second brightest $\mathrm{H}$ alpha knot in the NW complex (or NGC 4214-I) at groundbased resolution (see Fig. 2 in Maíz-Apellániz et al. 1999, where it is referred to as knot 5). As opposed to other bright $\mathrm{H}$ alpha knots, it has a relatively low value of [O III] $\lambda 5007 / \mathrm{H} \beta$ and also some $2-3$ arc sec. towards the SE there is a low-intensity $\mathrm{H} \alpha$ component redshifted by about $60 \mathrm{~km} \mathrm{~s}^{-1}$ (Maíz-Apellániz et al. 1999) which could now be interpreted as being caused by the SNR.

\section{The distance to NGC 4214}

To determine the distance to NGC 4214 we use the upper cut-off in the planetary nebula emission line luminosity function (PNLF) pioneered for Elliptical galaxies by Jacoby et al. (1989) and Jacoby et al. (1990), and developed to a high degree of sophistication in a long series of papers by Ciardullo and co-workers (Ciardullo et al. 2002, and references therein). The technique was shown to be also applicable to Magellanic Irrregulars (e.g. like NGC 4214) by Jacoby et al. (1990) who determined PNLF distance determinations for the LMC and SMC. It has also been successfully applied to other low-metallicity systems such as NGC 300 (Soffner et al. 1996) and NGC 55 (van de Steene et al. 2006). 
Fig. 4 The cumulative luminosity function of the planetary nebula candidates in the $[\mathrm{O} \mathrm{III}]$ and the $\mathrm{H} \alpha$ emission lines. These have a (raw) upper cutoff at

$\log F_{[\mathrm{O}}{ }_{\mathrm{III}}=-15.0 \pm 0.1$ and $\log F_{\mathrm{H} \alpha}=-15.4 \pm 0.1$, respectively. The systematic corrections that need to be applied to these cutoffs are discussed in the text

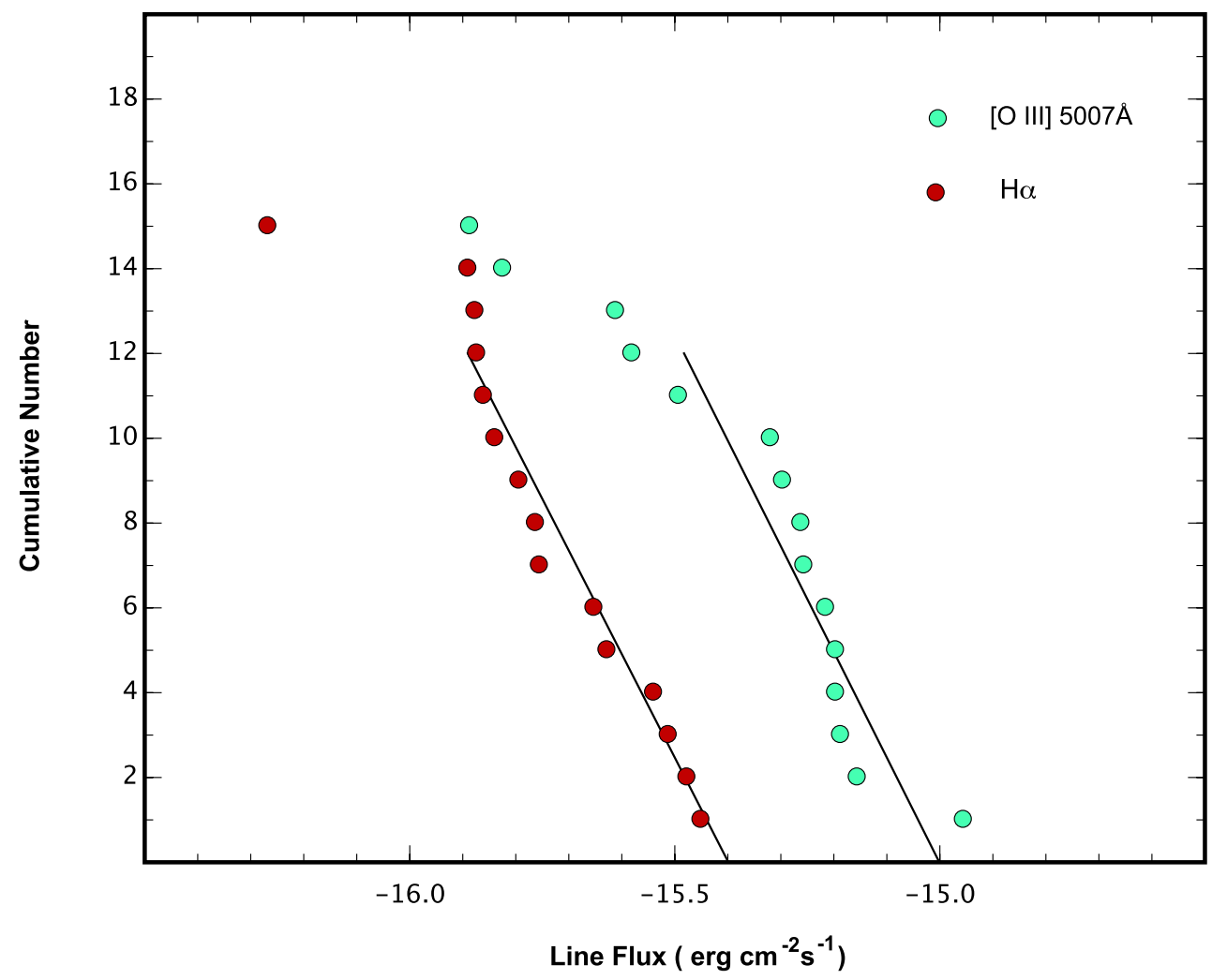

This method relies on the fact that the range of core mass of PN is very restricted, and has a sharply falling mass distribution at its upper end due to the strongly increasing importance of pre-PN mass-loss, and the intrinsic drop in numbers of stars with increasing mass as a consequence of the shape of the initial mass function (IMF). The emission line luminosity is directly related to the core-mass luminosity as the star evolves through the PN phase, but the rate of evolution across the HR Diagram accelerates strongly as the core mass is increased. For all of these reasons, the probability of finding a PN decreases very sharply with both increasing core mass and emission line luminosity. Thus, the upper cutoff in the luminosity function provides a standard candle, in much the same way as the tip of the RGB provides a standard candle in stars in a somewhat earlier stage of their evolution.

The errors intrinsic to this technique caused by varying stellar effective temperature and metallicity (which mostly affect the [O III] luminosity) were systematically investigated by Dopita et al. (1992). In Fig. 5 of that paper, they also gave observed absolute luminosity cutoffs in both [O III] and in $\mathrm{H} \beta$ for the Large and Small Magellanic Clouds (LMC and SMC). This is of particular relevance to NGC 4214, since this galaxy has an oxygen abundance $12+$ $\log (\mathrm{O} / \mathrm{H}$ ) in the range 8.2-8.4 (Kobulnicky and Skillman 1996) which puts it between the SMC $(12+\log (\mathrm{O} / \mathrm{H})=$ 8.05) and the $\operatorname{LMC}(12+\log (\mathrm{O} / \mathrm{H})=8.35)$ in metallicity (Russell and Dopita 1992). Thus, systematic effects of metallicity and stellar content can be avoided by making a estimate of the relative distances. This assumes that the absolute distances to the Magellanic Clouds have been well determined by independent techniques. The use of relative distance estimates also allows us to overcome issues connected with the shape of the upper PNLF function, since it obviates the need to build a theoretical PNLF, which is both theoretically complex, and subject to many uncertainties.

The measured fluxes of emission lines for these PN are given in Table 2. As can be seen, the relative faintness of the $\mathrm{H} \beta$ line means that errors in the flux determination are large, and in some objects, an $\mathrm{H} \beta$ flux could not be measured. For those $\mathrm{PN}$ for which both $\mathrm{H} \alpha$ and $\mathrm{H} \beta$ fluxes have been measured we find $\left\langle F_{\mathrm{H} \alpha} / F_{\mathrm{H} \beta}\right\rangle=3.41$. This is higher than the canonical Balmer Decrement (2.86) because of both reddening and the fact that $\mathrm{H} \alpha \mathrm{F} 657 \mathrm{~N}$ filter transmits both [N II] $\lambda \lambda 6548,6584$ emission lines as well as $\mathrm{H} \alpha$. The difference therefore provides an estimate of the mean value of the intensities of the [N II] lines relative to $\mathrm{H} \alpha$.

According to Burnstein and Heiles (1982) the HI data shows that galactic foreground extinction to NGC 4214 is effectively zero. However, Schlegel et al. (1998) used the $100 \mu \mathrm{m}$ far-IR dust emission to estimate $A_{B}=0.094 \mathrm{mag}$. We adopt this value to give foreground reddening estimates of $A_{5000 \AA}=0.08$ and $A_{6563 \AA}=0.06$ mag, respectively. We therefore apply a correction to the measured $\mathrm{H} \alpha$ flux of 0.854 to compensate for the contribution of the [N II] lines and internal extinction of the PN to the flux. 
The raw inferred cutoff PN luminosity in both the [O III] and $\mathrm{H} \alpha$ emission lines for NGC 4214 is shown in Fig. 4. Here we take a linear fit to the upper PNLF, in the same manner as was done in Dopita et al. (1992). However, these estimates have to be corrected for two systematic effects, First, the foreground dust extinction, must be taken into account. For [O III], the cutoff is measured to be $\left.\log F_{[\mathrm{O}} \mathrm{III}\right]=$ $-15.0 \pm 0.05$ which is increased to $\left.\log F_{[\mathrm{O}} \mathrm{III}\right]=-14.97 \pm$ 0.0.06, correcting for the Galactic foreground extinction of $A_{5000 \AA}=0.08$, while for $\mathrm{H} \alpha$, the cutoff is measured to be $\log F_{\mathrm{H} \alpha}=-15.4 \pm 0.05$ which is decreased to $\log F_{\mathrm{H} \alpha}=$ $-15.47 \pm 0.08$, correcting for the mean contribution of the [N II] lines to the measured flux. Finally, it is increased to $\log F_{\mathrm{H} \alpha}=-15.455 \pm 0.06$, correcting for the Galactic foreground extinction of $A_{6563 \AA}=0.06$.

Second, and more seriously, we need to apply a correction for the relative small number statistics of the PN in these plots. Ideally, for our method to provide an accurate distance the sample size should be doubled, or even tripled. We would almost certainly find more luminous PN. However, in a low mass galaxy such as NGC 4214, there are simply not enough PN. The effect of small sample size has been extensively discussed in the literature (see Ciardullo et al. (2002), and references therein). The analytical form of the luminosity function given by these authors suggests that the small number statistics of our observations can entrain a systematic error of up to 0.2 magnitudes, or $0.08 \mathrm{dex}$ in the cutoff luminosity-the true cutoff will be brighter. We therefore adopt upper luminosity cutoffs of $\left.\log F_{[\mathrm{O}}{ }_{\mathrm{III}}\right]=-14.89 \pm 0.10$ and $\log F_{\mathrm{H} \alpha}=-15.37 \pm 0.08$ for NGC 4214.

The luminosity cut-off in the LMC in [O III] is at $\left.\log F_{[\mathrm{O}} \mathrm{III}\right]=-11.31 \pm 0.03$ which is increased to $\log$ $F_{[\mathrm{O} \text { III }]}=-11.21 \pm 0.04$, correcting for the Galactic foreground extinction of $A_{V}=0.249$ (Schlegel et al. 1998). Therefore the ratio of distances of the two galaxies is $\log \left(D_{\mathrm{NGC} 4214} / D_{\mathrm{LMC}}\right)=1.84 \pm 0.14$. The same computation can be applied to the $\mathrm{H} \alpha$ fluxes. Assuming an intrinsic Balmer Decrement of 2.86, the luminosity cut-off in the LMC for $\mathrm{H} \alpha$ is at $\log F_{\mathrm{H} \alpha}=-11.94 \pm 0.02$. This is increased to $\log F_{\mathrm{H} \alpha}=-11.86 \pm 0.02$, correcting for the Galactic foreground extinction of $A_{R}=0.099$. This implies that the ratio of distances of the two galaxies is $\log \left(D_{\mathrm{NGC4214}} / D_{\mathrm{LMC}}\right)=1.75 \pm 0.10$. We will adopt the mean value of these two estimates, $\log \left(D_{\mathrm{NGC} 4214} / D_{\mathrm{LMC}}\right)=$ $1.795 \pm 0.12$.

Finally, the distance of the LMC has been critically reviewed by Walker (2003) and is given as $0.0506 \pm$ $0.0024 \mathrm{Mpc}$. This compares well with the mean distance from reddening independent techniques given in the NASA Extragalactic Database (NED); $0.051 \mathrm{Mpc}$. However there is a danger in uncritically using such a compilation see Schaefer (2008) for a warning. Ciardullo et al. (2002) has argued that the geometric distance given by Gould and Uza (1998) based on the analysis of the light echo of SN1987A is the most precise of all measurements, and Gould's distance is also favored by his PN Luminosity function measurements. In this case, the distance could be as little as $0.0472 \mathrm{Mpc}$. Here we will adopt the Walker (2003) distance to the LMC.

Therefore, taking the weighted mean of the [O III] and the $\mathrm{H} \alpha$ luminosity function cut-off data, we derive a distance to NGC 4214 (based on the NED distance of the LMC) of $3.16 \pm 0.5 \mathrm{Mpc}$. (This estimate may be reduced to as little as $2.9 \pm 0.4 \mathrm{Mpc}$ if the Gould and Uza (1998) or Ciardullo et al. (2002) distance to the LMC is adopted.)

Applying a similar analysis for the SMC PN, In [O III] the cutoff is at $\log F_{[\mathrm{O}}{ }_{\mathrm{III}}=-11.53 \pm 0.04$ which is increased to $\left.\log F_{[\mathrm{O}} \mathrm{II}\right]=-11.48 \pm 0.04$, correcting for the Galactic foreground extinction (Schlegel et al. 1998). This gives the ratio of distances of the two galaxies as $\log \left(D_{\mathrm{NGC} 4214} / D_{\mathrm{SMC}}\right)=1.705 \pm 0.14$. For $\mathrm{H} \alpha$ the SMC cutoff is at $\log F_{\mathrm{H} \alpha}=-11.93 \pm 0.03$ which is increased to $\log F_{\mathrm{H} \alpha}=-11.89 \pm 0.03$. Correcting for the Galactic foreground extinction (Schlegel et al. 1998), giving the ratio of distances of the two galaxies is $\log \left(D_{\mathrm{NGC} 4214} / D_{\mathrm{SMC}}\right)=$ $1.74 \pm 0.10$. We will adopt the weighted mean value of these two estimates, $\log \left(D_{\mathrm{NGC} 4214} / D_{\mathrm{LMC}}\right)=1.728 \pm 0.12$.

The distance of the SMC from Cepheids as given in NED is $0.0601 \mathrm{Mpc}$. Therefore, taking the mean of the [O III ] and the $\mathrm{H} \alpha$ luminosity function cut-off data, we derive a distance to NGC 4214 (based on the measured distance of the SMC) of $3.22 \pm 0.4 \mathrm{Mpc}$. Note that this is a little larger than the distance derived using the LMC as reference, and the difference between the two estimates is increased if the Gould and Uza (1998) LMC distance were to be adopted. From the average of the LMC and SMC estimates, we conclude that the PNLF technique gives a reddening independent distance to NGC 4214 of $3.19 \pm 0.36 \mathrm{Mpc}$.

Our distance estimate is larger, but hardly significantly, than the two recent estimates which used HST WFPC2 Iband data to determine the distance based on the tip of the Red Giant Branch method. Maíz-Apellániz et al. (2002) derived a value of $2.94 \pm 0.18 \mathrm{Mpc}$ while Drodovsky et al. (2002) obtained $2.7 \pm 0.3 \mathrm{Mpc}$. Very recently, Dalcanton et al. (2009) have used very deep F606W+F814W WFPC2 imaging to determine a more precise distance to the Galaxy from the tip of the red giant technique in a region roughly four arc. min from the center of the galaxy. They obtained $3.04 \pm 0.048 \mathrm{Mpc}$.

All these estimates based on HST data are smaller than earlier estimates based upon either the Blue Stragglers or the Tully-Fisher technique. Giving twice the weight to the MaízApellániz et al. (2002) estimation as either of the other two techniques (based upon the quoted errors), we conclude that the current best estimate distance to NGC 4214 is $2.98 \pm$ $0.13 \mathrm{Mpc}$. 


\section{Conclusions}

In this paper we have used HST Wide Field Camera 3 data to identify 17 planetary nebula candidates in NGC 4214. Of these, 15 are almost certainly bona fide $\mathrm{PN}$, and the remaining two are more likely to be extremely compact highexcitation $\mathrm{H}$ II regions. This number is just sufficient to establish a velocity-independent distance to the galaxy using the PN luminosity function. We have done this by determining the distance of NGC 4214 relative to the Magellanic Clouds. This avoids systematic effects due to metallicity and stellar population. The main remaining systematic error is caused by the small sample size, which would tend to overestimate the apparent distance to NGC 4214.

We have also identified seven supernova remnant candidates. Of these four are secure identifications, and these also display non-thermal spectra at radio wavelengths. We have also identified one strong young SNR candidate, with very strong oxygen emission lines. The remaining candidates are less secure, and these may simply be old recombining $\mathrm{H}$ II regions.

Acknowledgements This paper is based on observations with the NASA \& ESA Hubble Space Telescope obtained at the Space Telescope Science Institute, which is operated by the Association of Universities for Research in Astronomy, Incorporated, under NASA contract NAS5-26555. It uses Early Release Science observations made by the WFC3 Scientific Oversight Committee. We are grateful to the Director of the Space Telescope Science Institute for awarding Director's Discretionary time for this program. Dopita acknowledges the support of the Australian Research Council (ARC) through Discovery projects DP0984657 and DP0664434. This research has made use of the NASA/IPAC Extragalactic Database (NED) which is operated by the Jet Propulsion Laboratory, California Institute of Technology, under contract with the National Aeronautics and Space Administration. This research has also made use of NASA's Astrophysics Data System, and of SAOImage DS9 (Joye and Mandel 2003), developed by the Smithsonian Astrophysical Observatory.

\section{References}

Blair, W.P., Kirshner, R.P., Chevalier, R.: Astrophys. J. 2475, 879 (1981)

Blair, W.P., Long, K.S.: Astrophys. J. Suppl. Ser. 155, 101 (2004)

Burnstein, D., Heiles, C.: Astron. J. 87, 1165 (1982)

Chomiuk, L., Wilcots, E.M.: Astron. J. 137, 3869 (2009) (CW09)

Ciardullo, R., Feldmeier, J.J., Jacoby, G.H., Kuzio de Naray, R., Laychak, M.B., Durrell, P.R.: Astrophys. J. 577, 31 (2002)

D’Odorico, S., Benvenuti, P., Sabbadin, F.: Astron. Astrophys. 63, 63 (1978)

D’Odorico, S., Dopita, M.A., Benvenuti, P.: Astron. Astrophys. Suppl. Ser. 40, 67 (1980)

Dalcanton, J.J., et al.: Astrophys. J. Suppl. Ser. 183, 67 (2009)
Dopita, M.A., Blair, W.B., Long, K.S., et al.: Astrophys. J. 710, 964 987 (2010)

Dopita, M.A.: Astrophys. J. Suppl. Ser. 33, 437 (1977a)

Dopita, M.A.: Astrophys. J. 214, 179 (1977b)

Dopita, M.A., Meatheringham, S.J.: Astrophys. J. 367, 115 (1991a)

Dopita, M.A., Meatheringham, S.J.: Astrophys. J. 347, 21 (1991b)

Dopita, M.A., Vassiliadis, E., Wood, P.R., Meatheringham, S.J., Harrington, J.P., Bohlin, R.C., Ford, H.C., Stecher, T.P., Maran, S.P.: Astrophys. J. 474, 188 (1997)

Dopita, M.A., Jacoby, G.H., Vassiliadis, E.: Astrophys. J. 389, 27 (1992)

Drodovsky, I.O., Schulte-Ladbeck, R.E., Hopp, U., Greggio, L., Crone, M.M.: Astron. J. 124, 811 (2002)

Eck, C.R., Cowan, J.J., Branch, D.: Astrophys. J. 573, 306 (2002)

Fruchter, A., Sosey, M., et al.: The MultiDrizzle Handbook. STScI, Baltimore (2009). Version 3.0

Fruchter, A.S., Hook, R.N.: Publ. Astron. Soc. Pac. 114, 144 (2002)

Gordon, S.M., Kirshner, R.P., Long, K.S., Blair, W.P., Duric, N., Smith, R.C.: Astrophys. J. Suppl. Ser. 117, 89 (1998)

Gould, A., Uza, O.: Astrophys. J. 494, 118 (1998)

Jacoby, G.H., Ciardullo, R., Ford, H.C.: Astrophys. J. 339, 39 (1989)

Jacoby, G.H., Walker, A.R., Ciardullo, R.: Astrophys. J. 365, 471 (1990)

Joye, W.A., Mandel, E.: Astron. Data Anal. Softw. Syst. XII, 295, 489 (2003)

Kobulnicky, H.A., Skillman, E.D.: Astrophys. J. 471, 211 (1996)

Lasker, B.M.: Astrophys. J. 212, 390 (1977)

Long, K.S., Blair, W.P., Kirshner, R.P., Winkler, P.F.: Astrophys. J. Suppl. Ser. 72, 61 (1990)

Long, K.S., et al.: Astrophys. J. Suppl. Ser. (2010, submitted)

MacKenty, J.W., Maíz-Apellániz, J., Pickens, C.E., Norman, C.A., Walborn, N.R.: Astron. J. 120, 3007 (2000)

Maíz-Apellániz, J., Mas-Hesse, J.M., Muñoz-Tuñón, C., Vilchez, J.M., Castaneda, H.O.: Astron. Astrophys. 329, 409 (1998)

Maíz-Apellániz, J., Muñoz-Tuñón, C., Tenorio-Tagle, G., Mas-Hesse, J.M.: Astron. Astrophys. 343, 64 (1999)

Maíz-Apellániz, J., Cieza, L., McKenty, J.W.: Astron. J. 123, 1307 (2002)

Mathewson, D.S., Clarke, J.N.: Astrophys. J. 180, 725 (1973)

Mathewson, D.S., et al.: Astrophys. J. Suppl. Ser. 51, 345 (1983)

Mathewson, D.S., et al.: Astrophys. J. Suppl. Ser. 55, 189 (1984)

Mathewson, D.S., et al.: Astrophys. J. Suppl. Ser. 58, 197 (1985)

Mathewson, D.S., Healey: In: Kerr, F.J., Rodgers, A.W. (eds.) The Galaxy and the Magellanic Clouds, p. 283. The Australian Academy of Science, Canberra (1964)

Russell, S.C., Dopita, M.A.: Astrophys. J. 384, 508 (1992)

Schaefer, B.E.: Astron. J. 135, 112 (2008)

Schlegel, D.J., Finkbeiner, D.P., Davis, M.: Astrophys. J. 500, 525 (1998)

Soffner, T., Mendez, R.H., Jacoby, G.H., Ciardullo, R., Roth, M.M., Kudritzki, R.P.: Astron. Astrophys. 306, 9 (1996)

Ubeda, L., Maíz-Apellániz, J., MacKenty, J.W.: Astron. J. 133, 932 (2007)

Walker, A.R.: In: Alloin, D., Gieren, W. (eds.) Stellar Candles for the Extragalactic Distance Scale. Lecture Notes in Physics, vol. 635, p. 265 (2003)

van de Steene, G.C., Jacoby, G.H., Praet, C., Ciardullo, R., Dejonghe, H.: Astron. Astrophys. 455, 891 (2006)

Westerlund, B.E., Mathewson, D.S.: Mon. Not. R. Astron. Soc. 131, $371(1966)$ 